\title{
Single-Pulse Multi-Component Interferometric Rayleigh Scattering Velocimeter
}

\author{
Daniel Bivolaru, Paul M. Danehy, and Joseph W. Lee \\ NASA Langley Research Center, Hampton VA 23681, USA
}

Keywords: interferometer, Rayleigh scattering, interferogram processing, simultaneous measurement, multi-component, velocity.

\section{Summary}

Rayleigh scattering has previously been used for multi-component velocity measurements ${ }^{1}$. However, past work has generally been limited to excitation with continuous wave lasers resulting in a longer time response. We are developing a diagnostic system for time-resolved simultaneous measurement of multiple components of velocity in gas flow using a 10 -ns pulsed YAG laser. This system will be combined with Coherent anti-Stokes Raman spectroscopy (CARS) thermometry ${ }^{2}$ to allow simultaneous temperature and velocity measurements in supersonic combustion flows. Since we will use the same green laser beam for CARS and Rayleigh we will ensure that the temperature and velocity are measured at the same time and place. Dedicated optics collect the Rayleigh scattered light from different angles. This light is mixed together with the unshifted laser light before it is passed through a Fabry-Perot interferometer ${ }^{3}$. At the output of the interferometer, a high-sensitivity CCD camera images the interference fringe pattern. This pattern contains both the Rayleigh scattered signal from multiple collecting angles and the reference frequency information. Image processing software is used to analyze interferograms for Doppler shift to solve for velocity. Figure 1 shows three examples of interferograms containing frequency information to solve for one velocity component. Experiments were performed in stagnant air and a 3-mm diameter underexpanded jet flow. The jet had a total temperature of $300 \mathrm{~K}$ and a total pressure of about $4 \mathrm{~atm}$ and issued into ambient air at atmospheric pressure. We used a pulsed Nd:YAG laser at $10 \mathrm{~Hz}$ and $9 \mathrm{nsec}$, at 90 $\mathrm{mJ} / \mathrm{pulse}$. Measurements to date have included up to 2 velocity components simultaneously and a third component can be measured using the same method.

To improve light collection efficiency each part of the optical system was analyzed and optimized, including light collecting optics, light transmission through optical fibers, interferometer mirrors, and CCD camera. The use of lenses for collecting Rayleigh scattered light combined with an optical fiber for transmission, introduced photon loses about 15\%. More importantly, the number of photons transmitted by the interferometer input mirror was found to be low: 60 to one $(98 \%$ of the photons are rejected by the etalon). Pulse stretching of the investigation laser beam of up to $20 \mathrm{nsec}$ allowed an increase in the number of photons collected of up to 1.85 times.

The image processing methodology makes use of linearization of the concentric interference patterns from a planar Fabry-Perot interferometer and subsequent analysis ${ }^{3}$. The important variables are the center of the interferogram and the maximum fringe shift of the Doppler shifted signal. There are four steps of interferogram processing: 1 . The image center is found. 2 . The image is mapped by reading it in $r-\theta$ plane, where $r$ is the radius from the center and $\theta$ is the angle, using the original $(\theta, r)$ coordinate as the new $(x, y)$ coordinate. 3. The coordinates of the obtained data are rescaled in accordance to the linear pixel configuration of the CCD camera. 4. The fringe spacing is transformed by the radius relation $R_{p}+1=\left(R_{p}{ }^{2}\right.$ $+2 f 2 / k) 1 / 2$, between two consecutive rings of the Fabry-Perot interferogram, where $R$ is the radius of the ring, $p$ is the fringe number, $k$ is the order of interference, and $f$ is the focal length of the fringe forming lens. This step completed the conversion of the image, namely stretching data over concentric circles into data distribution over parallel lines of equal length. A fitting technique using multiple Gaussians is then applied to the transformed image to determine the free spectral range (FSR) and Doppler shift frequency $\Delta f$ for each data point. The velocity component is then calculated using the equation $\Delta f=\left(\mathbf{k}_{\mathrm{S}}-\mathbf{k}_{0}\right) V / \lambda$ where, $\lambda$ is the incident laser wavelength, $\mathbf{k}_{0}$ and $\mathbf{k}_{\mathbf{S}}$ are the incident and the scattered wave vectors, and $\boldsymbol{V}$ is the gas velocity.

Mixing the Rayleigh scattered light with the unshifted reference light minimizes the velocity measurement uncertainty by decreasing the adverse effects of the laser frequency drift, interferometer 
alignment errors, vibrations, and background light scattering. Figure 2 shows the precision of the technique is $11 \mathrm{~m} / \mathrm{s}$, based on 1 standard deviation over 50 consecutive measurements (at $10 \mathrm{~Hz}$ ) of one component of velocity in stagnant air. The instrument was configured for the velocity range of up to $2820 \mathrm{~m} / \mathrm{sec}$, so the resulting precision is less than $1 \%$ of the measurement range of the instrument. This precision can be improved if the set velocity range is decreased by decreasing the interferometer's free spectral range. However, in the present application where high-temperature flow is expected, the velocity range currently in use is appropriate. Measurements performed with this system in an atmospheric pressure flame show that the spectral peaks are resolvable and that signal-to-noise is sufficient for velocity measurement at temperatures $>1500 \mathrm{~K}$.

The method will be used to obtain accurate three component velocity measurements at high repetition rate $(20 \mathrm{~Hz})$ required to improve CFD computer models. In combination with a dual-pump CARS technique, this method will provide single-pulse multi-property measurement techniques for combustion research. The final paper will present details of the optical setup, image processing, measurements and error analysis.

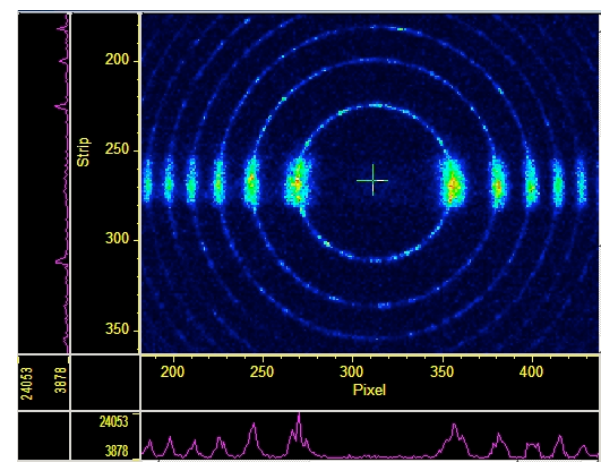

Fig. 1. Fabry-Perot interferogram showing the fringe pattern of reference and the Rayleigh scattered signal: interferogram of reference (concentric rings) and Rayleigh scattered light of zero Doppler shift obtained in stagnant air.

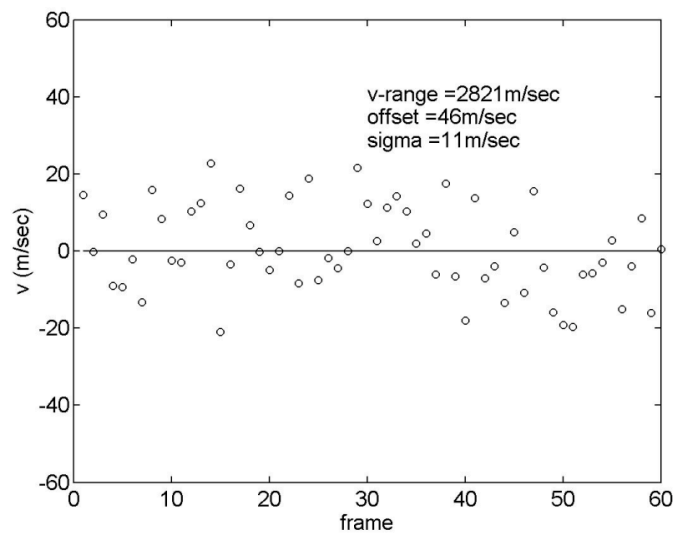

Fig. 2 Velocity measurements in stagnant air $(\mathrm{v}=0 \mathrm{~m} / \mathrm{s})$. The instrument showed a standard deviation of $11 \mathrm{~m} / \mathrm{s}$ and a zero-velocity offset of $46 \mathrm{~m} / \mathrm{s}$. The zero-velocity offset is repeatable and can be accounted for in the analysis.

\section{Acknowledgements}

This work was supported by The Office of The Secretary of Defense through the Test \& Evaluation / Science \& Technology Development Program, Hypersonic Test Focus Area, administered by Jere Matty at Arnold Engineering Development Center (AEDC). 


\section{References}

[1] Seasholtz, R.G., Goldman, L. J., "Three Component Velocity Measurements Using Fabry-Perot Interferometer", Second International Symposium on Applications of Laser Anemometry to Fluid Mechanics sponsored by 'Instituto Superior Tecnico (Lisbon)', Lisbon, Portugal, July 2-4, 1984 (also NASA TM 83692)

[2] Danehy, P.M., O’Byrne, S., Cutler, A.D., and Rodriguez, C. G., "Coherent anti-Stokes Raman Scattering (CARS) as a probe for supersonic hydrogen-fuel/air mixing," JANNAF APS/CS/PSHS/MSS Joint Meeting, Colorado Springs, Colorado, December 1-5, 2003.

[3] Bivolaru, D., Ötügen, M. V., Tzes, A. and Papadopoulos, G., "Image Processing for Interferometric Mie and Rayleigh Scattering Velocity Measurements, AIAA Journal, Vol. 37, No. 6, pp. 688-694, 1999. 


\title{
Single-pulse Multi-point Multi-component Interferometric Rayleigh Scattering Velocimeter
}

\author{
Daniel Bivolaru*, Paul M. Danehy ${ }^{\dagger}$, Joseph W. Lee, Richard L. Gaffney, Jr. ${ }^{\S}$, and Andrew D. Cutler ${ }^{\Uparrow}$ \\ NASA Langley Research Center, Hampton VA, 23681-2199
}

\begin{abstract}
A simultaneous multi-point, multi-component velocimeter using interferometric detection of the Doppler shift of Rayleigh, Mie, and Rayleigh-Brillouin scattered light in supersonic flow is described. The system uses up to three sets of collection optics and one beam combiner for the reference laser light to form a single collimated beam. The planar Fabry-Perot interferometer used in the imaging mode for frequency detection preserves the spatial distribution of the signal reasonably well. Single-pulse multi-points measurements of up to two orthogonal and one non-orthogonal components of velocity in a Mach 2 free jet were performed to demonstrate the technique. The average velocity measurements show a close agreement with the CFD calculations using the VULCAN code.
\end{abstract}

\section{Introduction}

$\mathrm{T}$ The gas flow velocity is an important parameter to measure in scientific, engineering and industrial applications. In aerodynamics, the velocity field dominates effects such as heat transfer and mass transport. Novel and improved methods of measuring velocity are sought for gases because existing methods do not work in all environments and/or require undesirable modifications to the flow, such as the need to seed particles or gasses into the flow. It is desirable to have a measurement system that can be used to determine flow properties, particularly velocity, but also temperature and density, in unseeded gas flows. This method should ideally work at both high and low temperatures, at multiple points, should measure multiple velocity components, and should be instantaneous. In this paper we present a variation of the Rayleigh/Mie scattering method that meets these requirements.

One approach to measure Rayleigh and Mie scattering spectra is to use atomic and molecular filters. In Doppler Global Velocimetry ${ }^{1,2}$ (DGV) two cameras view the flow, one of which looks through an Iodine filter $\left(I_{2}\right)$. If the laser frequency is kept constant, variations around this frequency due to Doppler shift correspond to variations in transmission. Using a calibration curve between transmissivity and velocity, up to three components of velocity can then be calculated. Although with the evolution of the seeding and computing techniques this method reached a high degree of accuracy, the principal limitation is that particles need to be seeded into flow. Only at low gas temperature can the DGV methodology be used in the Rayleigh scattering regime (scattering from molecules without seeding).

\footnotetext{
${ }^{*}$ NASA Postdoctoral Fellow, Advanced Sensing and Optical Measurement Branch, MS 493, AIAA Member

${ }^{\dagger}$ Research Scientist, Advanced Sensing and Optical Measurement Branch, MS 493, AIAA Associate Fellow

${ }^{\ddagger}$ Electro-optical Technician, Advanced Sensing and Optical Measurement Branch, MS 493, AIAA Member

$\S$ Aerospace Engineer, Hypersonic Air-breathing Propulsion Branch, MS 168, AIAA Senior Member

" Professor, The George Washington University, MAE Department, One Old Oyster Point Road, Suite 200, Hampton, Virginia, AIAA Associate Member
} 
Filtered Rayleigh Scattering ${ }^{3}$ (FRS) has been developed for unseeded measurement of velocity, temperature, and density in airflows and can be used for 2D mapping. The filter can also be used to suppress the background scattering. However, the $\mathrm{I}_{2}$ cell has limitations in that the absorption line from the spectrum that is used for filtering has a small bandwidth. Consequently, this approach has a low velocity dynamic range and does not work at high temperatures $(>1000 \mathrm{~K})$ when the Rayleigh spectrum is much broader than that of the filter.

A second approach permitting a larger dynamic range of measurable velocities and working at higher temperatures is the Interferometric Rayleigh Scattering method. This technique has previously been used for singlepoint velocity, density, and temperature measurements. Fabry-Perot and confocal etalons were used for Rayleigh signal analysis in conjunction with cooled CCD, intensified CCD, or photo-multiplier array that image the interferogram. ${ }^{4,5,6}$ Online referencing using a planar Fabry-Perot increased the precision of this method. ${ }^{7}$ Furthermore, it was shown that multiple points could be measured simultaneously with a Rayleigh scattering interferometric approach, but only one component of velocity was measured. ${ }^{8}$ This approach was used in some measurements without in-line referencing of the laser light, decreasing the instrument precision. On the other hand, in order to increase the number of photons collected this past work has generally been limited to excitation with continuous-wave lasers and integration for long times, resulting in single-point time-averaged measurements of velocity. Three velocity components have been measured simultaneously by using combined instruments of different types (Rayleigh and LDV) and having different methods of obtaining the velocity. ${ }^{9,10}$ This approach is very expensive, complicated and hard to use.

It has also been demonstrated that two orthogonal velocity components can be measured simultaneously at one point using one interferometer by reflecting back the probing laser beam ${ }^{11}$ or at multiple points by using a second optical cavity. ${ }^{12}$ However, this approach results in directional ambiguity of the flow velocity vector. Prior knowledge of the approximate magnitude and sign of the velocity components can remove this measurement ambiguity. The method described in Reference 12 removes the ambiguity by slightly detuning the secondary cavity.

This paper describes an optical system for gas velocity measurement using laser scattering from molecules or naturally-occurring particles analyzed for Doppler shift by a planar Fabry-Perot interferometer. Velocity measurements are performed simultaneously at multiple spatial locations. Multiple velocity components are measured. This system will be combined in the future with Coherent Anti-Stokes Raman Spectroscopy (CARS) thermometry ${ }^{13}$ to allow simultaneous temperature, species concentration, and velocity measurements in supersonic combustion flows.

The velocimetry system collects the Rayleigh scattered light from different angles. This light is mixed together with the unshifted laser light before it is passed through a planar Fabry-Perot interferometer for analysis. At the output of the interferometer, a high-sensitivity CCD camera images the interference fringe pattern. This pattern contains both the Rayleigh scattered spectrum from multiple collecting angles and the reference laser spectrum information. The shape of the scattering spectrum is function of the parameter $\boldsymbol{y}=\alpha \mathbf{K} a$, where $\alpha=p / \mu$ is the collision frequency ( $p$ is the gas pressure and $\mu$ is the shear viscosity) and $\mathbf{K} a$ is the acoustic spatial frequency ( $\mathbf{K}$ is the wave vector and $a$ is the speed of sound). For low density gases, when the parameter $\boldsymbol{y}<<1$, the Rayleigh spectrum can be approximated with a Gaussian shaped spectrum. The peak of the spectrum is Doppler shifted by a frequency proportional to the bulk velocity, the spectral width is proportional to the square root of the gas temperature, and its amplitude is proportional to the gas density. For higher density gases, a more detailed theoretical model is required to describe the Rayleigh scattering spectrum. As shown in Fig. 1, the Rayleigh spectrum for higher density gases, calculated using the Tenti S6 kinetic theory model, ${ }^{14}$ includes three Lorentzianshaped peaks in the spectral shape. To measure velocity, the Rayleigh spectrum is analyzed for Doppler shift applying a fitting technique and using the laser spectrum as reference. 


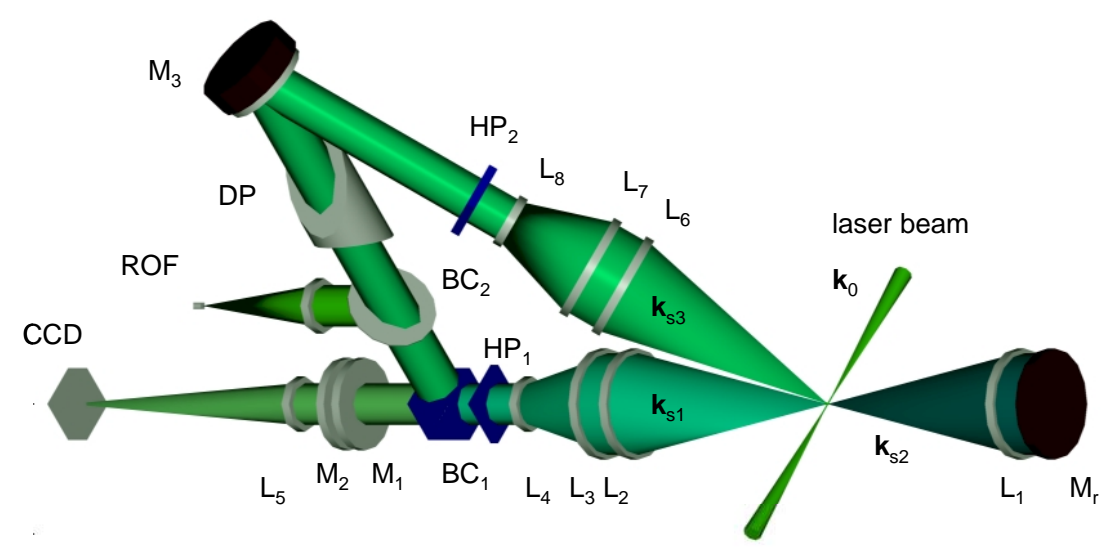

Figure 2. Experimental setup for simultaneous measurement of three components of velocity.

\section{Instrument Configuration}

\section{A. Collecting Optics and Scattering Geometry}

The optical setup of the instrument is illustrated in Fig. 2. The vertically polarized laser beam with the wave vector $\mathbf{k}_{0}$ is focused in a small region at the measuring location. The Doppler shift frequency of the scattered light collected in the direction of $\mathbf{k}_{\mathrm{s} 1}$ (perpendicular to $\mathbf{k}_{0}$ ) is given by $\Delta v_{1}=\left(\mathbf{k}_{\mathrm{s} 1}-\mathbf{k}_{0}\right) \cdot \mathbf{V}$, where the $\mathbf{k}_{\mathrm{s} 1}$ is the wave vector of the scattered light. The direction of $\left(\mathbf{k}_{\mathrm{s} 1}-\mathbf{k}_{0}\right)$ vector gives the direction of the velocity component $\mathrm{V}_{1}$ being measured. If a second collecting optics is placed along the same optical axis, but on the opposite side of the probed region, the scattering wave vector is $\mathbf{k}_{\mathrm{s} 2}=-\mathbf{k}_{\mathrm{s} 1}$, where $\left|\mathbf{k}_{\mathrm{s} 1}\right|=\left|\mathbf{k}_{\mathrm{s} 2}\right|=\left|\mathbf{k}_{0}\right|$. A mirror $\mathrm{M}_{\mathrm{r}}$ is used to reflect this collected light along the optical axis, sending it back to the interferometer. This light shows a Doppler shift $\Delta v_{2}=\left(\mathbf{k}_{\mathrm{s} 2}-\mathbf{k}_{0}\right)$. $\mathbf{V}$ and the velocity component $\mathrm{V}_{2}$ is measured along the vector $\left(\mathbf{k}_{\mathrm{s} 1}+\mathbf{k}_{0}\right)$, i.e. the two measured components are orthogonal. By slightly tuning the mirror $\mathrm{M}_{\mathrm{r}}$ in the vertical direction the image will contain two parallel lines situated symmetrically around the center of the interferogram. ${ }^{12}$ It will be shown that, each horizontal line represents one measurement direction.

The Rayleigh scattered light in the $\mathbf{k}_{\mathrm{s} 1}$ direction is collected and collimated by the $\mathrm{L}_{2}$ lens. The beam diameter is reduced to match the interferometer's input-aperture using lenses $\mathrm{L}_{3}$ and $\mathrm{L}_{4}$. In the $\mathbf{k}_{\mathrm{s} 2}$ direction, the light is collected and collimated by the lens $\mathrm{L}_{1}$ and reflected back toward the interferometer by the mirror $\mathrm{M}_{\mathrm{r}}$. The third component of velocity $\mathrm{V}_{3}$ with the wave vector $\mathbf{k}_{\mathrm{s} 3}$ is measured in a vertical direction in a plane containing $\mathbf{k}_{\mathrm{s} 1}$ and $\mathbf{k}_{\mathrm{s} 2}$ but perpendicular to the plane defined by the vectors $\mathbf{k}_{\mathrm{s} 1}$ and $\mathbf{k}_{0}$. The angle between $\mathbf{k}_{\mathrm{s} 1}$ and $\mathbf{k}_{\mathrm{s} 3}$ is about $33^{0}$. The light is collected and collimated by the lens $\mathrm{L}_{6}$, and the lenses $\mathrm{L}_{7}$ and $\mathrm{L}_{8}$ reduce the beam diameter. After the half wave plate $\mathrm{HP}_{2}$ rotates the beam polarization to horizontal, the mirror $\mathrm{M}_{3}$ reflects the collimated beam toward the polarization dependent beam combiner $\mathrm{BC}_{1}$. A Dove prism (DP) is used to rotate the laser beam image with an angle that is convenient for image processing (usually $90^{\circ}$ ). It can be positioned either on the $V_{1}$ (and $V_{2}$ ) or $V_{3}$ optical path. High quality achromatic lenses were used for collecting and collimating optics and $\lambda / 20$ flatness mirrors for $\mathrm{M}_{\mathrm{r}}$ and $\mathrm{M}_{3}$. Thereafter, the light is mixed together with laser light (used as reference frequency) before it is passed through the Fabry-Perot interferometer (F-P) using the beam combiner $\mathrm{BC}_{2}$.

At the output of the interferometer, a high-sensitivity CCD camera images the interference fringe pattern thus the probed region through the etalon. ${ }^{8}$ Therefore, the interferogram contains spatial as well as spectral information. Each set of fringes along the laser path in the image corresponds to a different measurement location in the flow. Because it is required that the light be collimated and perpendicular to the etalon input mirror $\mathrm{M}_{1}$, the field of view is restricted to a small region at the focusing point of lens $\mathrm{L}_{1}$. Dedicated imaging software processes each interferogram, extracting the Doppler shift of the Rayleigh/Mie signal relative to the reference laser frequency and determining the velocity values. An interferogram containing both the Rayleigh scattered signal (a straight vertical line pattern) and the reference laser frequency information (concentric rings) is shown in Fig. 4b. 
The air-gap adjustable planar interferometer was set for a free spectral range (FSR) either 3 or $5 \mathrm{GHz}$, depending on the velocity range to be measured. If the magnitude of the velocity of interest would have only positive or only negative values, as for example in the case of axial velocity, the instrument could use the full FSR for measurement without ambiguity. In turbulent regions, where the radial velocity can change sign, only half of the FSR can be used for measurement without ambiguity.

Due to the fact that our image recording technique employed on-line referencing, it wasn't necessary to use the interferometer temperature controller, neither was it required to take special care against vibrations. ${ }^{7,12}$

\section{B. Pulse Stretcher}

An increase in the number of photons collected by the receiving optics is desirable since Rayleigh scattering signals are low. This can be achieved in two ways: by decreasing the laser wavelength or by increasing the laser energy. In our experiments we have chosen to increase the number of photons up to 4 times by increasing the light source energy to $300 \mathrm{~mJ} /$ pulse using a Nd:YAG laser at $20 \mathrm{~Hz}$. The laser beam diameter was about $10 \mathrm{~mm}$ and we used an $\mathrm{f} / \# 4$ lens with the focal length of $400 \mathrm{~mm}$ to focus the beam at the measurement location. The breakdown of the gas limited the energy of the laser pulse at about $65 \mathrm{~mJ} /$ pulse for this configuration. To avoid this problem, the $10 \mathrm{nsec}$ pulse of the investigation laser beam was stretched to more than $40 \mathrm{nsec}$.

The optical arrangement of the pulse stretcher designed to obtain three consecutive laser pulses of almost identical amplitude is shown in Fig. 3. The system contains two beam splitters $\mathrm{BS}_{1}(68 \% \mathrm{~T})$ and $\mathrm{BS}_{2}(38 \% \mathrm{~T})$ and six identical flat mirrors $\mathrm{M}_{1-6}$ to create three delay lines of 0.3 , 1.0 , and $1.0 \mathrm{~m}$ respectively. The glass plates $\mathrm{GP}_{1}$ and $\mathrm{GP}_{2}$ are used to compensate for the change in the optical path by refraction of the laser beam that occurs at the beam splitters. A small portion of the vertically polarized light reflected from one of the compensating plates is collected by an optical fiber to be used as reference (ROF in Fig. 2). Two cross-polarizers (not shown) are used to adjust the reference intensity collected from a stray reflection from one of the compensating plates. At full input laser power, the pulse stretcher can deliver $210 \mathrm{~mJ} /$ pulse with an efficiency of about $70 \%$. Just over half of this energy is delivered to the probing volume because of losses in the transmission optics. The output beam pulse temporal shape differs slightly from the design shape shown in Fig. 3b. The tolerance of the commercially available beam splitters, and the setup constraint on the path lengths of the delay lines contributed to this difference.

\section{Beam Combining Configurations}

Figure 4 shows four possible combinations of mixing laser light and Rayleigh scattered light collected from different angles. The circular fringe pattern shown in Fig. 4a is generated by the reference laser frequency with no signal present. The vertical line in Fig. 4b, the image of the laser beam is used when multipoint measurement of one component of velocity $\left(\mathrm{V}_{1}\right)$ is desired. The positioning of the Rayleigh scattered light is symmetric on the interferogram and allows fast processing by averaging a small number of rows (reference) and columns $\left(\mathrm{V}_{1}\right)$, which pass through the center of the interferogram. In Fig. 4c the signal from two orthogonal velocity components, $\mathrm{V}_{1}$ (left) and $\mathrm{V}_{2}$ (right) are injected off axis in the vertical direction. A configuration as shown in Fig. $4 \mathrm{~d}$ with $\mathrm{V}_{1}$ (vertical on axis) and $V_{3}\left(135^{\circ}\right)$ can be used to measure two non-orthogonal velocity components. The advantage of Fig. 4d over $4 \mathrm{c}$ is potentially faster processing because the interferogram can be read directly along radii at angles of $0^{0}, 45^{\circ}, 90^{\circ}$ and $135^{\circ}$. Figure $4 \mathrm{e}$ shows a combination of two orthogonal velocity components in the horizontal 


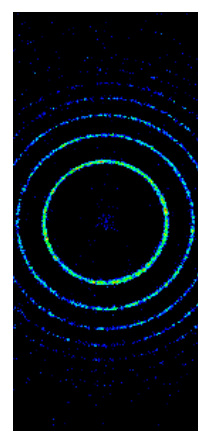

(a)

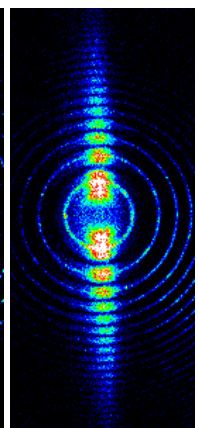

(b)

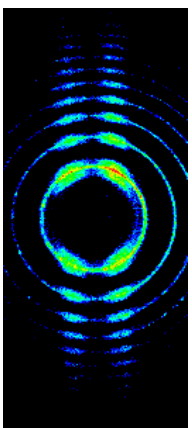

(c)

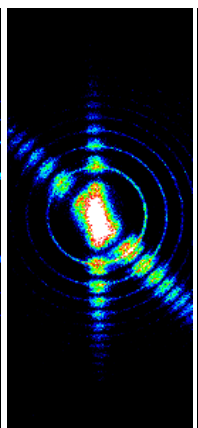

(d)

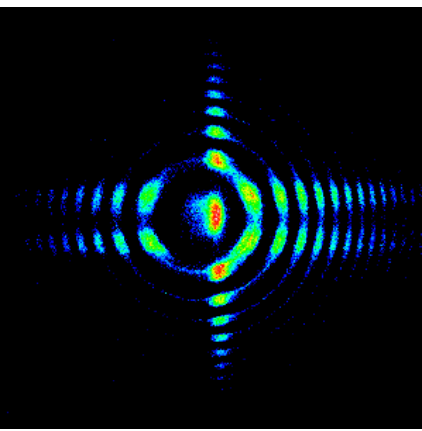

(e)

Figure 4. Combining on the same interferogram Rayleigh scattered light from different angles with reference laser light: (a), reference laser light; (b), multipoint one component of velocity; (c), and (d) multipoint two components of velocity; (e), multipoint three components of velocity.

direction $\left(\mathrm{V}_{1}\right.$ and $\left.\mathrm{V}_{2}\right)$ and one non-orthogonal in the vertical direction $\left(\mathrm{V}_{3}\right)$. Since in the case of an interferogram of type (c), and (e) the interferogram must be processed entirely, the processing time is considerably higher than in (b) and (d).

Although the intensity of the reference laser light must be monitored carefully to be of the order of the received scattered light, the system does not require special devices for filtering this radiation. As will be shown later, referencing the scattered light with unshifted laser light in a single interferogram reduces the velocity measurement uncertainty.

\section{Image Processing}

Image processing software is used to process and analyze the entire interferogram for the Doppler shift of the Rayleigh signal and the measured free spectral range (distance between consecutive ring patterns), and to solve for velocity. The image processing methodology consists of linearization of the concentric interference patterns from a planar Fabry-Perot interferogram and subsequent analysis. The important variables are the center of the interferogram, the free spectral range of the interferometer, and the maximum fringe shift of the Doppler shifted signal. An interferogramprocessing example of a pattern containing the reference laser frequency information (concentric rings, as shown in Fig. 4a) is shown in Fig. 5. There are four steps: 1 . The image center is found. 2 . The image is mapped by reading it in $r-\theta$ plane, where $r$ is the radius from the center and $\theta$ is the angle, using the original $(r, \theta)$ coordinate as the new $(x, y)$ coordinate (the resulting image is shown in Fig. 5a). 3. The coordinates of the obtained data are rescaled in accordance to the linear pixel configuration of the CCD camera. For small reading angles $\theta$, the conversion described in steps 2 and 3 can be simplified by averaging a low number of lines or columns symmetrically along the interferogram diameter. 4. The fringe spacing is transformed by the radius relation $R_{p+1}$ $=\left(R_{p}{ }^{2}+2 f 2 / k\right)^{1 / 2}$ between two consecutive rings (a)

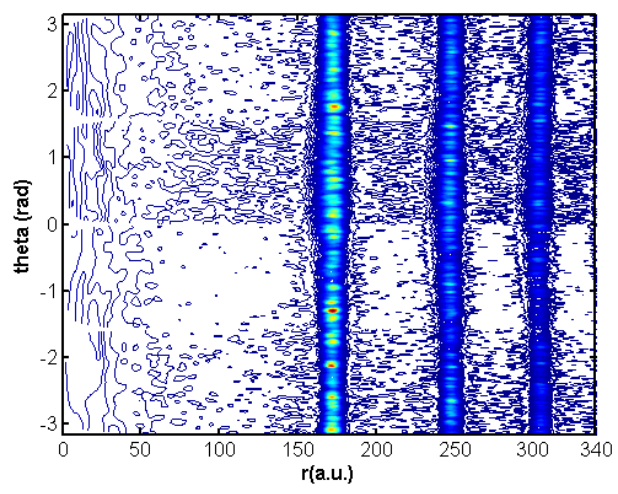

(b)

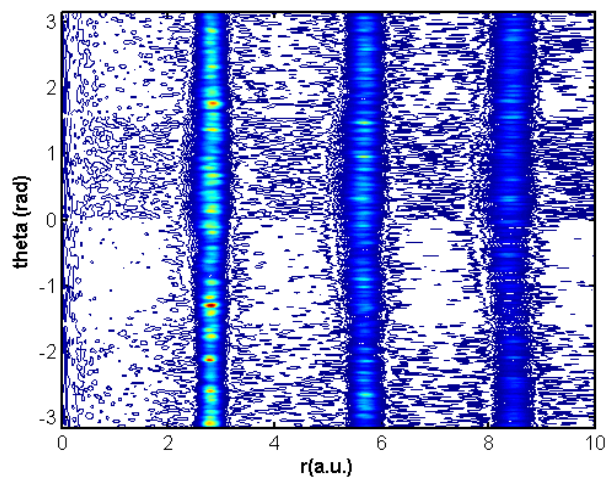

Figure 5. Transformation of concentric interference patterns from a Fabry-Perot interferometer (Fig. 4a) to a) parallel lines, and b) parallel lines of equal spacing spectra. 

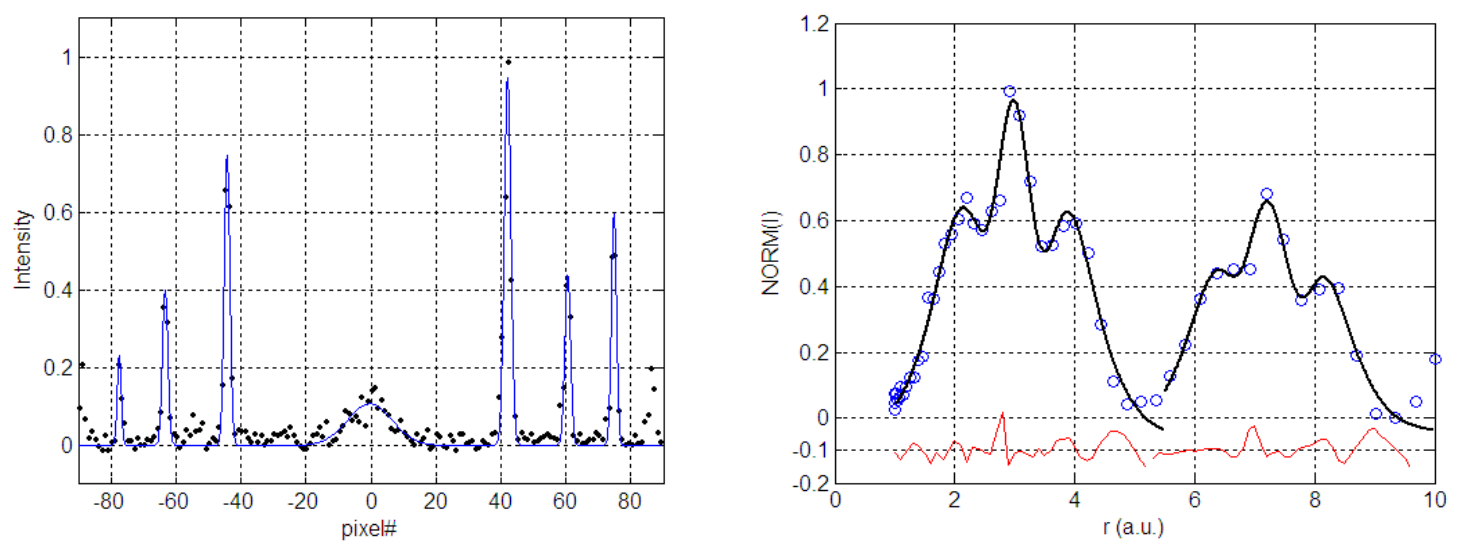

Figure 6. a) Fabry-Perot spectrum of reference laser light, and b) two linearized Rayleigh-Brillouin spectra of low Doppler shift. The spectra of Mie or Rayleigh scattering for $y<<1$ are similar with the Gaussian shape spectrum shown in (a). The RMS in Fig. b) shows the goodness of fit when forcing three Lorentzians and one Gaussian as the fit function.

of the Fabry-Perot interferogram, where $R$ is the radius of the ring, $p$ is the fringe number, $k$ is the order of interference, and $f$ is the focal length of the fringe forming lens. This step completes the conversion of the image, namely stretching data over concentric circles into data distributed over parallel lines of equal length. The final image, Fig. 5b can be processed directly in 2D by autocorrelation, ${ }^{7}$ or by binning the lines in a single average spectrum around a chosen angle $\theta$ and applying a fitting technique. In the present work, a fitting technique using as a function of the flow conditions, multiple Gaussians for Rayleigh and Mie scattering ${ }^{12}$ (Fig. 6a), and a combination of Lorentzians and Gaussians for Rayleigh-Brillouin scattering (Fig. 6b), was applied to the linearized spectrum to determine the free spectral range (FSR) and Doppler shift frequency $\Delta f$ for each fringe. The velocity component was then calculated knowing the experimental geometry and using the equation $\Delta f=\left(\mathbf{k}_{\mathrm{s}}-\mathbf{k}_{\mathbf{0}}\right) \boldsymbol{V} / \boldsymbol{\lambda}$ where, $\lambda$ is the incident laser wavelength, $\mathbf{k}_{\mathbf{0}}$ and $\mathbf{k}_{\mathrm{s}}$ are the incident and the scattered wave vectors, and $\boldsymbol{V}$ is the gas velocity.

\section{Velocity Measurement}

\section{A. Mach 2 Cold Jet}

Experiments to demonstrate the technique in a noisy environment were carried out in a small-scale Mach 2 jet facility at NASA Langley Research Center. The jet was formed with a convergent-divergent nozzle designed by the method of characteristics to produce a supersonic flow of $\mathrm{M}=2$ for a $\gamma=1.3$ ( $\mathrm{M}$ is the Mach number of the flow and $\gamma$ is the perfect gas constant). As designed, it had a throat diameter of $7.51 \mathrm{~mm}$ and an exit diameter of $10 \mathrm{~mm}$; it was subsequently found that, due to manufacturing error, the throat diameter was $7.66 \mathrm{~mm}$ (a fact significant in that the CFD work described below used the "as-designed" contour). The jet discharged into the atmosphere. No special care was taken for drying or filtering neither the air supply nor the ambient air. Naturally occurring Mie scattering from water clusters condensation was present only in the mixing layer region. Although the system can measure both Mie and Rayleigh scattering by adjusting the CCD camera gain, this need for adjustment made it difficult to obtain a continuous scan across the jet diameter. A camera with automatic gain control or with a larger dynamic range would address this problem.

The jet was mounted on a 2D stage, allowing axial and radial scans of the jet at the maximum speed of 0.55 $\mathrm{mm} / \mathrm{sec}$ in increments of $0.1 \mathrm{~mm}$. The instantaneous shadowgraph images of the flow (approximately $1 \mu \mathrm{sec}$ exposure time) taken at different stagnation chamber pressure and temperature values are presented in Fig. 7 . Three experiments were performed with nozzle plenum pressures of 5.5, 8.6 and $13.6 \mathrm{~atm}$ and plenum temperature of about $285 \mathrm{~K}$. This corresponds to an over-expanded case, a perfectly expanded case and an under-expanded case. We probed a volume of about $1.5 \mathrm{~mm}$ length and $0.2 \mathrm{~mm}$ diameter perpendicular to the flow direction. The spatial resolution of each measurement point was about $0.2 \times 0.2 \times 0.2 \mathrm{~mm}^{3}$. The minimum distance from the nozzle exit that could be measured was at about $0.5 \mathrm{~mm}$ from the exit plane. Since the interferogram contains spatial as well as spectral information, the separation distance between the first two consecutive fringe orders on one side of the 
interferogram corresponds to about $0.2 \mathrm{~mm}$ in the flow and the diameter of the first order fringe to about $0.5 \mathrm{~mm}$ in the flow.

For comparison, numerical simulations of the experimental flow field were performed using the VULCAN CFD code. This program solves the discretized Navier-Stokes equations using a structured-grid finite-volume formulation of the governing equations. ${ }^{15}$ Inviscid fluxes were computed using Roe's upwind flux difference split scheme and the turbulence was modeled with the K-omega model of Wilcox. The gas was assumed to be calorically perfect with a ratio of specific heats of 1.4. Three calculations were performed using the pressures and temperatures in the plenum chamber identical to those from the experiment. The grid used in the calculations started in the plenum upstream of the nozzle throat and extended 61 nozzle-exit diameters downstream of the nozzle exit. In the radial direction the outer boundary was more than 40 nozzle-exit diameters from the centerline. There were 609 grid points between the nozzle exit and the downstream outflow boundary, with 401 of those points in the first 5 nozzle-exit diameters downstream of the nozzle exit. This produced a streamwise grid spacing of $0.05 \mathrm{~mm}$ at the nozzle exit and a grid spacing of $0.2 \mathrm{~mm}$ at a 0.5 exitdiameters downstream of the nozzle exit. There were 801 points in the radial direction between the centerline and the outer boundary. At the nozzle exit, there were 161 evenly-spaced points between the centerline and one nozzle radius, giving a radial grid spacing of $0.03125 \mathrm{~mm}$. In order to reduce numerical difficulties associated with solving a jet flowing into quiescent air, a slight pressure gradient was imposed across the external domain. This, along with the entrainment from the jet produced an external flow of approximately $7 \mathrm{~m} / \mathrm{sec}$. The radial (v) and axial (u) velocity distribution of the over-expanded jet using these calculations is presented in Fig. 8. The coordinate $\mathrm{x}$ is in the streamwise direction and $\mathrm{y}$ in the radial direction of the jet with the origin situated axially in the nozzle exit plane.

\section{B. Radial velocity along jet axis}

The interferogram configuration shown in Fig. 4b was chosen for this measurement. In one test the jet was scanned axially in the streamwise direction at constant speed while recording interferograms at the laser pulse frequency of $20 \mathrm{~Hz}$ for 90 seconds. To compute the radial flow velocity ( $\left.\mathrm{V}_{1}\right)$, five rows were averaged through the center of the interferograms. This data was converted from the spatial domain (pixel) to the frequency domain as described earlier and in more detail in reference [9]. One spectrum processed in this way is shown in Fig. 6b. A combined function of one Gaussian and three Lorentzians were fitted to this data to determine with sub-pixel resolution the location of the peaks and implicitly the frequency. The low amplitude peaks in each spectrum are the sidebands of the Rayleigh-Brillouin spectra (see also the theoretical spectrum of Fig. 1). The two peaks shown in Fig. $6 \mathrm{~b}$ (corresponding to the left side of the interferogram) show the simultaneous measurement at two locations in the flow situated at about $0.2 \mathrm{~mm}$ apart. Similarly, there are two locations on the right side of the interferogram, up to a total of four points in each image. The spectrum of the reference frequency (laser frequency) used to identify the zero-velocity fringe location (extracted from a perpendicular direction in respect to the previous spectrum) was fitted 
with Gaussian functions (Fig. 6a). A series of 1000 interferograms (8000 spectra) were processed in this way. Fig. 9 shows the average of the radial velocity measured simultaneously at four points on the jet axis. The mean of the measured FSR was calculated using all data and its variation around the mean was converted to velocity as if it would be a Doppler shifted signal. The result was plotted with an offset of -300 $\mathrm{m} / \mathrm{sec}$ in the same figure for comparison. This fluctuation in the measured FSR was found to be less than $1 \%$ of the full range of the instrument. Translated in velocity, this corresponds to a standard deviation of about $25 \mathrm{~m} / \mathrm{sec}$.

Because the measurement volume was off axis by about $0.5 \mathrm{~mm}$, the radial velocity in the streamwise direction is not zero. The data shows the variation in velocity when the measurement volume passes through the shock structure. Considering that the effect of turbulence was minimal in the region investigated, the velocity spikes seen in the plot are mainly due to the errors introduced by the on-line fitting method.

For comparison, an average of the radial velocity at four points of the measurement volume scan was extracted from the CFD data. The result was plotted against the measured data and is shown in the Fig. 9 as a red line. Although the measured data shows a change from maximum to zero velocity faster than the CFD data in the expansion region of the cell structures at about 5 and $18 \mathrm{~mm}$ from nozzle, the two sets follow a similar trend.

\section{Axial velocity at two points near centerline}

Static measurements of velocity were performed using the type of interferogram shown in Fig. 4e. A region on the jet axis at 2.5 nozzle diameters upstream of the nozzle was selected for this experiment. The interferometer was set for a FSR of $5 \mathrm{GHz}$ allowing a velocity measurement up to about $3500 \mathrm{~m} / \mathrm{sec}$ without ambiguity. Four hundred interferograms containing information to compute three components of velocity were recorded at a laser pulse frequency of $20 \mathrm{~Hz}$ and analyzed as described earlier. The histogram of the measured axial velocity $\left(\mathrm{V}_{3}\right)$ at two locations, $\mathrm{u}_{2}$ and $\mathrm{u}_{3}$ situated $0.5 \mathrm{~mm}$ apart is presented in Fig. 10.

The velocity fluctuations of about $35 \mathrm{~m} / \mathrm{sec}$ are mainly due to the errors introduced by the on-line fitting method. Although the experimental noise of the facility was substantial, the simultaneous calculation of the reference reduced the errors introduced by vibrations detuning the interferometer to a minimal value. As before, the variation in the measured FSR (plotted around the mean as zero velocity) was found to be smaller than $1 \%$ of the full range of the instrument. Converted in velocity this uncertainty is about $25 \mathrm{~m} / \mathrm{sec}$. The uncertainty might possibly be caused by the errors in fitting the speckle-dominated reference. A potential enhancement would be to improve the reference light source and to use more elaborate fitting techniques of the Rayleigh-Brillouin spectra, which would take into account the interferometer transfer function and the intensity distribution of the combined input beam. Another way to improve the fitting technique precision is to increase the number of photon counts by increasing the laser energy or by increasing the photon efficiency of the camera. A more careful selection of the receiving optics, for an increased interferogram resolution and an increased number of photons per pixel received, would also help decrease these errors.

The small difference in the measured velocity values at these two locations demonstrates the system's capability for simultaneous
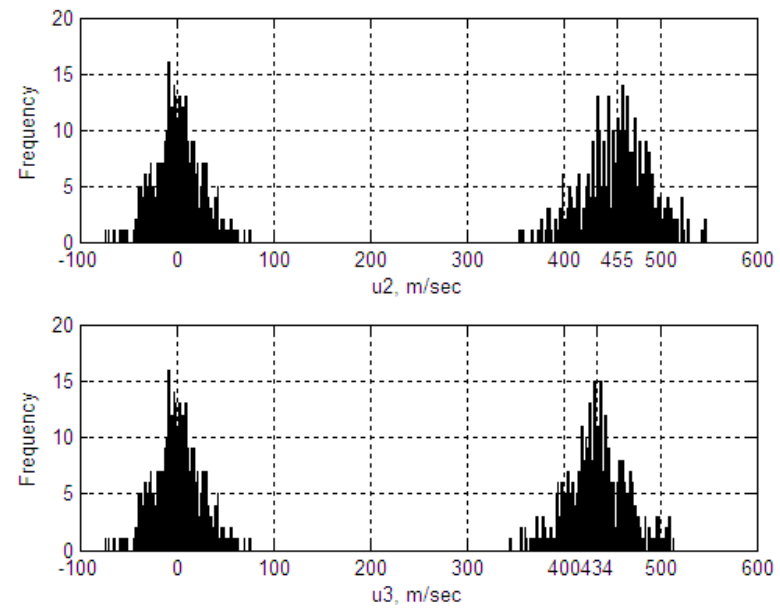

Figure 10. Instantaneous measurement of axial velocity at $x=25 \mathrm{~mm}$ on the axis of a Mach 2 over-expanded jet at $P_{0}=5.5 \mathrm{~atm}$ and $T_{0}=286.5 \mathrm{~K}$. The two-point measurement of $u_{2}$ and $u_{3}$ is simultaneous. 
measurement with high spatial resolution. In principle, the next outer fringe patterns can also be analyzed for velocity, but with reduced resolution. The average velocity at these two locations is about $5 \%$ higher than the value obtained by the CFD calculations, which is $422 \mathrm{~m} / \mathrm{sec}$. This discrepancy may be caused by uncertainty in the location of the measurement.

\section{Conclusion}

A new technique using a single Fabry-Perot interferometer that enabled simultaneous, multiple-point measurements of up to three components of velocity was demonstrated. The velocity was determined by analyzing the interferogram containing spectral as well as spatial information. The mixing of the laser light and background scattered light with the Rayleigh scattered light in the same interferogram was used to reduce the velocity measurement uncertainty by simultaneous post processing. The adverse effects of the laser frequency drift, interferometer alignment errors, vibrations, and background light scattering were minimized by the use of this method. The laser energy at the measuring volume was increased about 4 times by pulse stretching, while keeping the energy per pulse under the limit of the gas breakdown.

To approximate the Rayleigh-Brillouin spectrum, we used a combined function of one Gaussian and three Lorentzians in our fitting process to the measured data. For this system we showed that the fluctuations in the measured velocity (less than $25 \mathrm{~m} / \mathrm{sec}$ ) are mainly due to uncertainties in fitting the reference spectra.

By determining slightly different velocity values at different locations in the flow, we demonstrated the system's capability for simultaneous measurement with high spatial resolutions.

With a few improvements, the technique is capable of obtaining accurate three-component velocity data at the 20 $\mathrm{Hz}$ repetition rate required to improve CFD models. In combination with a dual-pump CARS technique, this method will provide single-pulse multi-property measurements for combustion research.

\section{Acknowledgments}

We wish to express our appreciation to Mr. George Rumford, program manager of the Defense Test Resource Management Center's (DTRMC) Test and Evaluation/Science and Technology (T\&E/S\&T) program, for funding of this effort under the Hypersonic Test focus area. We acknowledge the help of Keith .D. Grinstead and Steve Jones for laboratory work and Lloyd Wilson for running the supersonic jet facility. Also, we wish to thank Jim Meyers at NASA Langley Research Center for invaluable discussions and for the use of his laboratory and equipment.

\section{References}

${ }^{1}$ Komine, H., Brosnan, S.J., Litton, A. H., and Stappaerts, E. A., “Real-Time Doppler Global Velocimetry,” AIAA-91-0337, 29th Aerospace Sciences Meeting, Reno, NV, January 1991.

${ }^{2}$ Meyers J.F. 'Development of Doppler global velocimetry as a flow diagnostics tool,' Meas. Sci. Technol. 6, pp.769-783. 1995.

${ }^{3}$ Miles, R., B., Lempert, W., and Forkey, J., N., "Laser Rayleigh Scattering,” Review Article, Measurement Science and Technology, Vol. 12, No., pp. 33-51, 2001.

${ }^{4}$ Seasholtz, R.G., Zupac, F. J., Schneider, S. J., "Spectrally resolved Rayleigh scattering diagnostic for hydrogen-oxygen rocket plume studies” AIAA-1991-462, 29th Aerospace Sciences Meeting, Reno, NV, January, 1991.

${ }^{5}$ Seasholtz, R.G., Panda, J., "Rayleigh Scattering Diagnostic for Dynamic Measurement of Velocity and Temperature," AIAA-99-0641, 37th Aerospace Sciences Meeting, Reno, NV, 1999.

${ }^{6}$ Seasholtz, R. G. \& Panda, J. "Rayleigh Scattering Diagnostic for Simultaneous Measurements of Dynamic Density and Velocity,” AIAA-2000-0642, 38th Aerospace Sciences Meeting, Reno, NV, January 2000.

${ }^{7}$ Bivolaru, D., Ötügen, M. V., Tzes, A. and Papadopoulos, G., "Image Processing for Interferometric Mie and Rayleigh Scattering Velocity Measurements”, AIAA Journal, Vol. 37, No. 6, pp. 688-694, 1999.

${ }^{8}$ Seasholtz, R.G., "Instantaneous 2D Velocity and Temperature Measurements in High Speed Flows Based on Spectrally Resolved Molecular Rayleigh Scattering”, AIAA 33rd Aerospace Sciences Meeting, Reno, AIAA-95-0300, January 9-12, 1995.

${ }^{9}$ Seasholtz, R.G., Goldman, L. J., "Combined Fringe and Fabry-Perot Laser Anemometer for Three Component Velocity Measurements in Turbine Cascade Facility”, AGARD Conference Proceedings No.399, Advanced Instrumentation for Aero Engine Components, Presented at the Propulsion and Energetics Panel $67^{\text {th }}$ Symposium, Philadelphia, PA, USA, pp. 13-1 to 13-15, 19-23 May 1986.

${ }^{10}$ Seasholtz, R.G., Goldman, L. J., “Three Component Velocity Measurements Using Fabry-Perot Interferometer”, Second International Symposium on Applications of Laser Anemometry to Fluid Mechanics sponsored by 'Instituto Superior Tecnico (Lisbon)', Lisbon, Portugal, July 2-4, 1984 (also NASA TM 83692) 
${ }^{11}$ Eggins, P. L., and Jackson, D. A.,“A two-component laser-Doppler anemometer using a single Fabry-Perot interferometer,” J. Phys. D: Appl. Phys., Vol. 8, pp. 45-47, 1975.

${ }^{12}$ Bivolaru, D. Danehy, P. M., Lee, J. W.,“ Intracavity Rayleigh/Mie scattering for multi-point two-component velocity measurement," Optics Letters, Submitted for publication.

${ }^{13}$ Danehy, P.M., O’Byrne, S., Cutler, A.D., and Rodriguez, C. G., "Coherent anti-Stokes Raman Scattering (CARS) as a probe for supersonic hydrogen-fuel/air mixing," JANNAF APS/CS/PSHS/MSS Joint Meeting, Colorado Springs, Colorado, December 1-5, 2003.

${ }^{14}$ Tenti G., Boley, C. D., and Desay, R. C., “On the kinetic model description of Rayleigh-Brillouin scattering from molecular gases,” Can. J. Phys, Vol. 52, pp. 285, 1974.

${ }^{15}$ White, J. A. and Morrison, J. H., "A Pseudo-Temporal Multi-Grid Relaxation Scheme for Solving the Parabolized NavierStokes Equations," AIAA paper 99-3360, June, 1999. 